\title{
Relación entre tiempos de alimentación, composición nutricional del desayuno y estado nutricional en estudiantes universitarios de Valparaíso, Chile
}

\section{Relationship between feeding schedule, nutritional composition of breakfast and nutritional status among university students in Valparaíso, Chile}

\section{RESUMEN}

Se han descrito estilos de vida poco saludables en estudiantes universitarios, como omisión del desayuno, alto consumo de comida rápida y horarios dispersos. El objetivo de este trabajo fue relacionar distintas características de la ingesta alimentaria con el estado nutricional en este grupo. Se realizó un estudio observacional analítico, donde participaron 130 jóvenes sedentarios. Se evaluó el estado nutricional y la ingesta alimentaria. Un 25\% de la muestra presentó malnutrición por exceso (IMC), mientras que el $50 \%$ presentó exceso de grasa corporal. Los hombres con exceso de grasa corporal mostraron un porcentaje de adecuación significativamente menor en el aporte energético al desayuno $(p=0.021)$ y los sujetos (ambos sexos) con exceso de grasa corporal presentaron un porcentaje de adecuación también menor en la ingesta calórica al almuerzo en comparación con los sujetos con bajas reservas energéticas ( $p=0.004), y$ su vez, todos los sujetos consumían un exceso de energía en la merienda, independiente de su estado nutricional. Se observó también una relación dependiente entre horarios de alimentación y el porcentaje de grasa corporal $(p=0.044)$. Una baja ingesta energética al desayuno junto con una alta ingesta durante la merienda con intervalos de ayuno mayores a 4 horas al menos una vez al día, podrían estar asociados a un exceso de grasa corporal en estudiantes universitarios sedentarios.

Palabras clave: Composición corporal; Desayuno; Estado nutricional, Estudiantes universitarios; Horarios de alimentación.

\footnotetext{
ABSTRACT

Unhealthy lifestyles, such as omission of breakfast, high intake of fast food, and lack of mealtime schedules, have been described among university students. The aim of this study was to relate different characteristics of food intake with the nutritional status of university students. An analytical observational study was conducted among 130 sedentary young people. Nutritional status (weight, height and body fat percentage) and food intake (24h food recall and eating habits survey) were assessed. One quarter of the sample had overweight or obesity (BMI), while 50\%
}

Cristina Concha', Gabriela González', Raúl Piñuñuri², Carina Valenzuela ${ }^{1 *}$.

\footnotetext{
1. Escuela de Nutrición y Dietética, Facultad de Farmacia, Universidad de Valparaíso, Valparaíso, Chile. 2. Escuela de Nutrición y Dietética, Facultad de Salud, Universidad Bernardo O'Higgins, Santiago, Chile.
}

\begin{abstract}
*Dirigir correspondencia a: Carina Valenzuela A. Escuela Nutrición y Dietética. Facultad de Farmacia, Universidad de Valparaíso. Gran Bretaña 1193, Playa Ancha, Valparaíso, Chile. CP 2360102. Email: carina.valenzuela@uv.cl
\end{abstract}

Este trabajo fue recibido el 17 de abril de 2018. Aceptado con modificaciones: 18 de diciembre de 2018. Aceptado para ser publicado: 07 de enero de 2019.

had excess body fat. Men with excess body fat had a lower energy intake at breakfast $(p=0.021)$ and the subjects (both sexes) with excess body fat had a lower calorie intake at lunch compared to subjects with low energy reserves ( $p=$ 0.004). Moreover, all subjects consumed excess energy at teatime, independent of body composition. A dependent relationship between feeding schedules and body fat percentage was observed $(p=0.044)$. Low energy intake at breakfast along with a high intake during teatime, with fasting intervals of more than 4 hours between meals at least once a day, could be associated with excess body fat in sedentary university students.

Key words: Body composition; Breakfast; Mealtime schedules; Nutritional state; University students. 


\section{INTRODUCCIÓN}

El inicio del periodo universitario suele coincidir con el final de la adolescencia y el paso a la edad adulta; y frecuentemente es el momento en que los estudiantes asumen la responsabilidad de su alimentación por primera vez. Distintos factores contribuirán en el establecimiento de nuevos hábitos alimentarios, que en muchos casos son mantenidos a lo largo de la vida'. En esta población se han observado patrones alimentarios y estilos de vida poco saludables, como bajo nivel de actividad física, irregularidad en el patrón de comidas, consumo elevado de comida "rápida" y de bebidas azucaradas y alcohólicas, seguimiento de dietas nutricionalmente inadecuadas con baja densidad nutricional, alta incidencia de trastornos de la conducta alimentaria, entre otras ${ }^{2,3}$.

Uno de los tiempos de comidas fundamentales para distribuir de forma adecuada la ingesta calórica durante el día es el desayuno. Garaule et al. ${ }^{4}$ han sugerido que individuos que no acostumbran a desayunar pueden tener un mayor consumo de calorías durante las horas de tarde/ noche. Otros autores observaron ${ }^{5}$, a través de un estudio aleatorizado realizado en 93 mujeres con exceso de peso, que un desayuno alto en calorías con una ingesta reducida en la cena es benéfico y puede ser una alternativa útil para el manejo de la obesidad y síndrome metabólico.

Shafiee et al. ${ }^{6}$ reportaron que el desayuno es un factor protector para el desarrollo de síndrome metabólico y obesidad. Adicionalmente, en un estudio transversal se evaluó el consumo de energía mediante encuesta de Recordatorio $24 \mathrm{~h}$ distribuyendo horarios del mediodía (11.00 am a 17.00 pm) y tarde (17.00 pm a $00.00 \mathrm{am})$, en 99 individuos, se mostró que los participantes que consumían $\geq 33 \%$ de su consumo diario de energía al medio día fueron significativamente menos propensos a tener sobrepeso $u$ obesidad, y aquellos que consumían $\geq 33 \%$ de la ingesta energética diaria en la noche eran dos veces más propensos a tener sobrepeso u obesidad ${ }^{7}$. Otras investigaciones avalan que las personas que comen mayormente por la noche son más propensas a aumentar de peso y tienen menor capacidad para perderlo.

De hecho, un retraso del ritmo circadiano se ha asociado con alteraciones metabólicas y obesidad ${ }^{8,9}$. No solo la ingesta del desayuno se ha asociado con un mejor estado nutricional, sino que también la composición nutricional de éste. Se ha descrito que un mayor consumo de carbohidratos al desayuno se relaciona significativamente con un menor Índice de Masa Corporal (IMC), y por el contrario saltarse el desayuno o consumir un desayuno alto en grasa y bajo en fibra está asociado con un mayor IMC ${ }^{10}$. Mientras, otros autores reportaron que un desayuno alto en proteínas y grasas puede tener beneficios metabólicos en pacientes con diabetes mellitus $2^{11}$.

En base a estos antecedentes, el objetivo del presente estudio fue relacionar la distribución y aporte nutricional del desayuno, número de comidas al día y horarios de alimentación con el estado nutricional según IMC y porcentaje de grasa corporal en jóvenes universitarios sedentarios de la Universidad de Valparaíso.

\section{MATERIALES Y MÉTODOS}

Estudio transversal y analítico realizado en 130 estudiantes de la Universidad de Valparaíso. El protocolo del estudio fue aprobado por el comité de bioética de la Facultad de Farmacia de la Universidad de Valparaíso.

\section{Participantes}

La muestra por conveniencia (no probabilística) incluyó a estudiantes entre 18 y 30 años pertenecientes a la Facultad de Farmacia, Facultad de Ciencias, Facultad de Ciencias del Mar y Facultad de Ingeniería Civil Industrial de la Universidad de Valparaíso.

La muestra fue seleccionada considerando los siguientes criterios: (a) Criterios de inclusión: Universitarios de carrera diurna de la quinta región pertenecientes a la Universidad de Valparaíso, sedentarios (ejercicio físico menor a 3 veces por semana y duración menor a 30 minutos por vez), con participación voluntaria y sin previa intervención nutricional, con firma de carta de consentimiento informado y cuestionario de hábitos alimentarios resuelto y (b) Criterios de exclusión: Universitarios que estaban bajo tratamiento nutricional, sedentarios temporales (secundario a discapacidad física temporal, por ejemplo fractura), con diagnóstico de enfermedad crónica con relevancia nutricional con tratamiento dietoterapéutico, y/o que no hayan contestado las encuestas requeridas.

A los sujetos, posterior al consentimiento informado por escrito, se les aplicó la Encuesta de Recordatorio 24 horas para evaluar su alimentación habitual correspondiente a un día de semana y un día de fin de semana, con ayuda de imágenes de porciones de alimentos de Chile y medidas caseras. Se evaluó el estado nutricional de los individuos a través de las mediciones de peso y porcentaje de grasa corporal, para lo cual se utilizó una balanza marca Tanita modelo BF-679W. Los sujetos fueron ubicados en el centro de la plataforma, distribuyendo el peso en ambas piernas, brazos al costado, con un mínimo de ropa y descalzos. Para medir la talla se utilizó un estadiómetro portátil para adultos marca SECA modelo 220. Los individuos se midieron de pie, erguidos y con los brazos al costado, con los talones juntos y adosados a la superficie posterior del estadiómetro. La cabeza en plano de Frankfurt, con espalda alta y glúteos apoyados en tabla vertical del estadiómetro.

Posteriormente se clasificó el estado nutricional de los individuos en bajo peso, normal, sobrepeso y obesidad, con los siguientes puntos de corte para IMC $<18,5,18,5-24,9$, $25-29,9$ y $>30$, respectivamente. El porcentaje de grasa corporal se clasificó como adecuado en rangos de $20-30 \%$ y $12-20 \%$ para mujeres y hombres respectivamente, cualquier valor fuera del rango se consideró como inadecuado ${ }^{12}$.

Procesamiento de Datos

Para determinar la composición nutricional del desayuno 
y la distribución calórica de los diferentes tiempos de comida, se estableció la recomendación de energía a través de la ecuación propuesta por la FAO/OMS $2004^{13}$, donde se calculó la tasa metabólica basal para cada individuo según estado nutricional bajo peso, normal, sobrepeso y obesidad utilizando: peso mínimo aceptado, peso real, ideal y ajustado respectivamente. Posteriormente se incorporó el factor de actividad física para los individuos sedentarios correspondiente a 1,4.

Para el análisis cuantitativo de los datos de la encuesta de recordatorio de 24 horas se estandarizaron los gramos de cada alimento con la tabla de Porciones de intercambio y la Composición química de los alimentos de la pirámide chilena $^{14}$ y posteriormente se ingresaron al programa nutricional Food Processor (Food Processor SQL ${ }^{\circledR}$, ESHA Research, Salem, OR, USA). El análisis cualitativo de la alimentación de los sujetos se realizó considerando los tiempos de alimentación, horarios de alimentación, frecuencia de consumo de desayuno y distribución calórica en los distintos tiempos de comida. La clasificación de las variables se detalla a continuación.

Tiempos de alimentación: 1) Regulares: Corresponde a la realización de las 4 comidas recomendadas (Desayuno, almuerzo, merienda, cena); 2) Irregulares: Corresponde a la omisión de una o más de las 4 comidas recomendadas.

Horarios de alimentación: 1) Regulares: Corresponde a intervalos entre comidas menores o iguales a 4 horas; 2) Irregulares: Corresponde a intervalos entre comidas mayor a 4 horas, en al menos un tiempo de comida.

Frecuencia de consumo del desayuno: 1) Normal: 6-7 días/semana; 2) Regular: 3 - 5 días /semana; 3) Rara vez: 0 - 2 días /semana

Esta variable se utilizó para determinar la Ingesta de Desayuno: 1) Si consume: Sujetos con una frecuencia Normal del desayuno; 2) No consume: Sujetos con frecuencia Regular o Rara vez.

Se categorizó de esta manera ya que se quiere saber qué sujetos tienen como un hábito permanente la ingesta del desayuno.

\section{Distribución calórica}

Corresponde a la distribución de las calorías durante el día, se considera adecuado los siguientes rangos según tiempo de comida: 1) Desayuno: 20-25\%; 2) Almuerzo: 30- 35\%; 3) Merienda: 15 - 20\%; 4) Cena: $20-25 \%$.

Los alimentos ingeridos fuera de estos tiempos de comida se clasificaron en: 1) Colación: Alimentos con horario establecido y consumidos de manera habitual; 2) Snack: Alimento sin horario establecido y consumido cuando hay apetito; 3) Ambos se considerarán sólo para calcular el total de energía diaria consumida.

\section{Análisis estadístico}

Se utilizó el programa IBM SPSS Statistics versión 22. Se aplicó el test de Kolmogorov-Smirnov para verificar la normalidad de la distribución de la muestra en las variables cuantitativas. Para comparar las variables cualitativas se utilizó la prueba de $\mathrm{Chi}^{2}$, mientras que para las variables cuantitativas con distribución normal, se utilizó ANOVA de una vía y Tukey. Para aquellas sin distribución normal, se utilizó Kruskall-Wallis. Las variables cuantitativas se expresan como promedio \pm desviación estándar (DE). El nivel de significancia utilizado fue de $5 \%$ para todos los casos.

\section{RESULTADOS}

La muestra en estudio estuvo constituida por 130 universitarios sedentarios de la Universidad de Valparaíso, donde el $71,5 \%$ correspondió a mujeres (93 personas) y un $28,5 \%$ fueron hombres (37 personas), con una edad promedio de 20,9 $\pm 1,9$ años. Según IMC, un 3,1\% presentó bajo peso, $71,5 \%$ estado nutricional normal y $25,4 \%$ exceso de peso ( $20,8 \%$ sobrepeso y $4,6 \%$ obesidad). En cuanto al peso y la talla promedio de las mujeres fue de $60,2 \pm$ $10,6 \mathrm{~kg}$ y 1,61 $\pm 0,05 \mathrm{~m}$ respectivamente, para los hombres fue de 70,1 $\pm 10,2 \mathrm{~kg}$ y $1,72 \pm 0,05 \mathrm{~m}$, respectivamente (Tabla 1).

Con respecto al porcentaje de grasa corporal (Figura 1) se observó que el $50 \%$ de la muestra presentó exceso de grasa corporal $(\% G C>20$ en hombres y \%GC>30 en mujeres), mientras que según clasificación por IMC sólo un $35 \pm 4,7 \%$ de las mujeres y un $23,5 \pm 2,8 \%$ de los hombres presentaron malnutrición por exceso.

En relación al número de comidas realizadas, el 63,1\% consumía 3 comidas diarias (Figura 2A) y el $90 \%$ de la muestra presentó tiempos de comidas irregulares, es decir, omitían al menos una de las 4 comidas principales del día

Tabla 1.

\begin{tabular}{|lcccc|}
\hline Estado nutricional & $\begin{array}{c}\text { Cajacterísticas de la muestra } \\
\text { Bajo peso } \\
\mathbf{X} \pm \mathbf{D E}\end{array}$ & $\begin{array}{c}\text { Normal } \\
\mathbf{X} \pm \mathbf{D E}\end{array}$ & $\begin{array}{c}\text { Sobrepeso } \\
\mathbf{X} \pm \mathbf{D E}\end{array}$ & $\begin{array}{c}\text { Obesidad } \\
\mathbf{X} \pm \mathbf{D E}\end{array}$ \\
\hline Edad (años) & $20,8 \pm 2,6$ & $20,9 \pm 1,9$ & $21,3 \pm 2,2$ & $20,3 \pm 1,0$ \\
Talla (mt) & $1,60 \pm 0,04$ & $1,64 \pm 0,08$ & $1,67 \pm 0,08$ & $1,63 \pm 0,06$ \\
Peso $(\mathrm{kg})$ & $45,6 \pm 2,7$ & $59 \pm 7,8$ & $76,6 \pm 8,9$ & $84,8 \pm 4,2$ \\
IMC (kg/mt $\left.{ }^{2}\right)$ & $17,8 \pm 0,4$ & $22 \pm 1,6$ & $26,9 \pm 1,2$ & $32 \pm 1,4$ \\
\hline
\end{tabular}




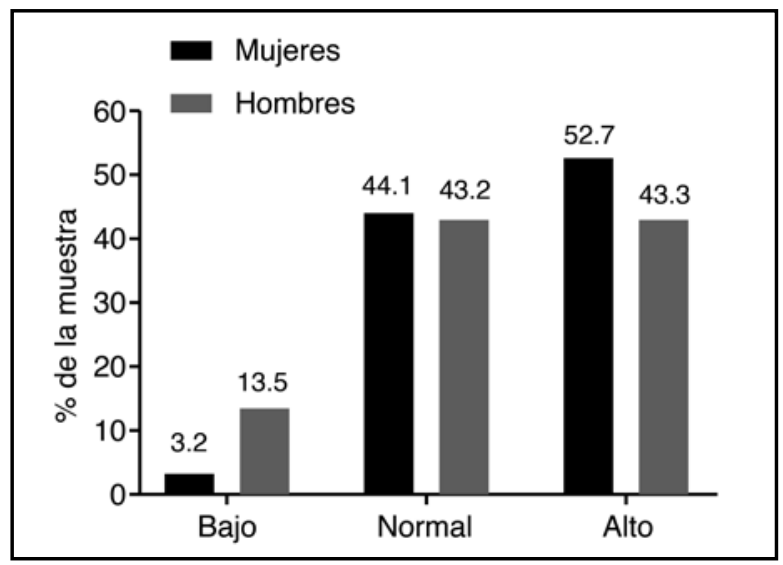

Tabla 2. Caracterización nutricional del desayuno según sexo.

\begin{tabular}{|lcc|}
\hline Sexo & $\begin{array}{c}\text { Mujer } \\
\text { X } \pm \text { DE }\end{array}$ & $\begin{array}{c}\text { Hombre } \\
\text { X } \pm \text { DE }\end{array}$ \\
\hline Energía (kcal) & $261 \pm 154$ & $297 \pm 240$ \\
Distribución calórica (\%) & $18 \pm 10,2$ & $15 \pm 11,9$ \\
Proteínas (g) & $10,6 \pm 8,2$ & $11,4 \pm 10,4$ \\
Hidrato de carbono (g) & $42,9 \pm 24,1$ & $46 \pm 38,4$ \\
Lípidos (g) & $6,4 \pm 6,5$ & $8,3 \pm 8,1$ \\
& & \\
\hline
\end{tabular}

Figura 1. Distribución del porcentaje de grasa corporal según sexo.

\section{A: $\mathrm{N}^{\circ}$ de comidas}

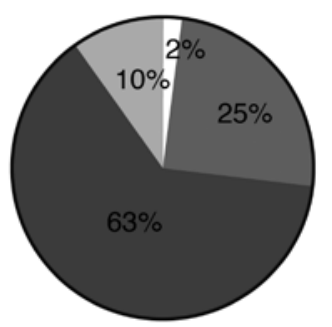

\section{B: Horarios de alimentacion}

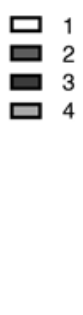

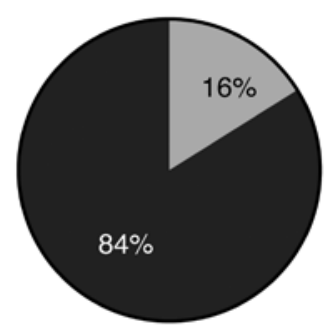

$\square$ Horarios regulares

Horarios irregulares

Figura 2. Porcentaje de individuos según número de comidas y horarios de alimentación.

(Desayuno-Almuerzo-Merienda-Cena). Esto también se refleja en los horarios de alimentación, puesto que un $84 \%$ de los sujetos en estudio poseía horarios irregulares, con intervalos entre comidas de más de 4 horas de ayuno en al menos una ocasión al día, y sin colaciones establecidas (Figura 2B).

En cuanto a la caracterización nutricional del desayuno (Tabla 2), se observó que el aporte energético es muy variable en ambos sexos, aunque la media no superó las
300 kcal. Con respecto a la distribución calórica diaria del desayuno, pocos individuos tuvieron una ingesta adecuada según lo recomendado (20-25\% del total de calorías diarias), el $43 \%$ de las mujeres alcanzó una ingesta adecuada y sólo el $30 \%$ de los hombres, según sus requerimientos individuales. Además, ambos sexos mantuvieron una distribución homogénea de los macronutrientes, los cuales se ordenaron de manera decreciente en hidratos de carbono, proteínas y lípidos.

Tabla 3. Aporte nutricional del desayuno asociado al porcentaje de grasa corporal en hombres.

\begin{tabular}{|lcccc|}
\hline & Bajo & Normal & Exceso & Valor p \\
\hline${\text { Energía }(\mathrm{kcal})^{1}}^{1}$ & $417 \pm 156$ & $461 \pm 189$ & $364 \pm 124$ & 0,446 \\
Proteínas $(\mathrm{g})^{\mathrm{y}}$ & $18,2 \pm 5,2$ & $17,4 \pm 10,7$ & $13,1 \pm 5,7$ & 0,458 \\
Hidrato de carbono $(\mathrm{g})^{1}$ & $61,1 \pm 40,9$ & $72,9 \pm 28,7$ & $56,3 \pm 15,8$ & 0,407 \\
${\text { Lípidos }(\mathrm{g})^{2}}_{\text {Distribución calórica (\%) }}$ & $11,8 \pm 4,6$ & $12,6 \pm 7,2$ & $10,5 \pm 9,0$ & 0,695 \\
& $19,8 \pm 8,1^{\mathrm{a}}$ & $19,3 \pm 13,1^{\mathrm{a}}$ & $8,9 \pm 10,2^{\mathrm{b}}$ & 0,021 \\
\hline
\end{tabular}

Datos presentados como Media \pm DE.

${ }^{1}$ ANOVA de una vía.

${ }^{2}$ Prueba Kruskall-Wallis ${ }^{a, b}$ : Prueba HDS de Tukey, letras diferentes indican diferencias significativas $(p<0,05)$. 
Tabla 4. Porcentaje de adecuación de la ingesta calórica diaria según sexo y estado nutricional.

\begin{tabular}{|lcccccc|}
\hline \multirow{2}{*}{ Estado nutricional } & Bajo peso & \multicolumn{2}{c}{ Normal } & \multicolumn{2}{c|}{ Sobrepeso } & Obesidad \\
\cline { 2 - 6 } & Mujeres & Hombres & Mujeres & Hombres & Mujeres & Mujeres \\
\hline Recomendación de energía $(\mathrm{kcal})$ & 1670 & 2349 & 1851 & 2395 & 1874 & 2058 \\
Ingesta diaria de energía $(\mathrm{kcal})$ & $1548 \pm 636$ & $2094 \pm 735$ & $1389 \pm 417$ & $1896 \pm 503$ & $1446 \pm 351$ & $1457 \pm 137$ \\
Adecuación (\%) & $93 \pm 0,4$ & $89 \pm 0,3$ & $92 \pm 0,3$ & $78 \pm 0,2$ & $77 \pm 0,2$ & $71 \pm 0,1$ \\
& & & & & & \\
\hline
\end{tabular}

Tabla 5. Horarios de alimentación según porcentaje de grasa corporal.

\begin{tabular}{|lcc|}
\hline $\begin{array}{l}\text { Porcentaje de } \\
\text { grasa corporal }\end{array}$ & $\begin{array}{c}\text { Horarios de alimentación } \\
\text { (frecuencia, \%) } \\
\text { Regular }\end{array}$ & Irregular \\
\hline Normal & 24.6 & 75.4 \\
Exceso & 10.8 & 89.2 \\
Total & 17.2 & 82.8 \\
& & \\
\hline
\end{tabular}

Prueba $\mathrm{Chi}^{2} \mathrm{p}=0,044$

No se encontró una relación de dependencia entre la ingesta de desayuno y estado nutricional de los sujetos (datos no se muestran, $\mathrm{p}=0,167$ ). Aun así, se observó un alto porcentaje de individuos con exceso de peso que no consumen desayuno. De igual manera ocurrió en el caso del porcentaje de grasa corporal (datos no se muestran, $p=$ $0,471)$. Con respecto al aporte nutricional del desayuno asociado al estado nutricional y porcentaje de grasa corporal para ambos sexos, no hubo diferencias significativas para ninguna variable. Sin embargo, se observó una baja ingesta energética en los individuos obesos según IMC y con exceso de grasa corporal, además de una baja distribución calórica del desayuno con respecto a lo recomendado
(20-25\%), en comparación a los individuos normales (datos no se muestran). Particularmente, en el caso de hombres no se observaron diferencias para el consumo de energía, proteínas, hidratos de carbono y lípidos según porcentaje de grasa corporal, sin embargo, sí respecto a la distribución calórica, representando así el desayuno un menor porcentaje de la ingesta diaria en varones con exceso de grasa corporal $(p<0,05)$ (Tabla 3). Del mismo modo, al observar la adecuación de la ingesta total diaria los sujetos con sobrepeso y obesidad presentaron una menor ingesta que los sujetos normales (Tabla 4).

Se encontró una asociación entre los horarios de alimentación y el porcentaje de grasa corporal, donde los sujetos con exceso de grasa en su mayoría presentan horarios de alimentación irregulares $(p<0,05)$ (Tabla 5), esta asociación no se encontró con el estado nutricional según IMC.

Se observó una diferencia en el porcentaje de adecuación del almuerzo según porcentaje de grasa corporal para la muestra total (Tabla 6), con una menor ingesta energética en relación a lo recomendado en los sujetos con exceso de grasa corporal en este tiempo de comida en comparación con aquellos con bajos depósitos adiposos, lo que se mantiene para las mujeres $(64.7 \%$ vs $114.7 \%$; $p<0,05$, datos no se muestran). De la misma forma, en los hombres se observaron diferencias ( $p<0,05$, datos no se muestran) en el porcentaje de adecuación del desayuno, con una

Tabla 6. Porcentaje de adecuación por tiempo de comida asociado a porcentaje de grasa corporal.

\begin{tabular}{|lcccc|}
\hline & $\begin{array}{c}\text { Bajo } \\
\mathbf{X} \pm \mathbf{d e}\end{array}$ & $\begin{array}{c}\text { Normal } \\
\mathbf{X} \pm \mathbf{d e}\end{array}$ & $\begin{array}{c}\text { Exceso } \\
\mathbf{X} \pm \mathbf{d e}\end{array}$ & Valor $\mathbf{p}$ \\
\hline Desayuno (\%) & $76,8 \pm 26,0$ & $64,9 \pm 43,9$ & $54,4 \pm 37,0$ & 0,173 \\
Almuerzo (\%) & $105 \pm 24,8^{\text {a }}$ & $76,6 \pm 29,3^{\mathrm{b}}$ & $68,4 \pm 30,8^{\mathrm{b}}$ & 0,004 \\
Merienda $(\%)^{1}$ & $117 \pm 49,8$ & $118 \pm 60,5$ & $117 \pm 57,7$ & 0,994 \\
Cena $(\%)^{2}$ & $7,6 \pm 21,6$ & $18,1 \pm 39,5$ & $10,0 \pm 28,6$ & 0,367 \\
\hline
\end{tabular}

Datos presentados como Media \pm DE.

'Prueba ANOVA de una vía

${ }^{2}$ Prueba Kruskall-Wallis

a,b Prueba HDS de Tukey, letra indica diferencias significativas $(p<0,05)$ 
menor ingesta energética en relación a lo recomendado en los sujetos con exceso de grasa corporal en comparación con aquellos con depósitos adiposos bajos y normales (32.8\% vs $81.8 \%$ y $71.2 \%$, respectivamente). En general, los sujetos con exceso de grasa corporal tuvieron una baja y posiblemente insuficiente ingesta de energía durante las primeras comidas del día y una ingesta energética excesiva según lo recomendado en horario nocturno.

\section{DISCUSIÓN}

En el presente estudio la mayor parte de la muestra presentó un estado nutricional normal según IMC (71,5\%), mientras que un 25,4\% de los individuos presentó exceso de peso, lo que entrega un escenario más optimista que el nacional, considerando que la última Encuesta Nacional de Salud, en el rango de 20 a 29 años, reportó sólo un 37,7\% de sujetos con estado nutricional normal ${ }^{15}$. Por otra parte, se observó que un 50\% de los sujetos en estudio presentaron exceso de grasa corporal, dentro de los cuales un 24,6\% eran sujetos con estado nutricional normal según IMC. De acuerdo a lo descrito en la literatura, el IMC tiene una mejor concordancia con el porcentaje de grasa en sujetos con obesidad ${ }^{16}$, de forma que a determinados valores "normales" el IMC parece no identificar adecuadamente a los sujetos con riesgo ${ }^{17}$, como se observó en este estudio. Es relevante destacar la necesidad de incluir el porcentaje de grasa corporal como indicador más específico.

Con respecto a los tiempos de alimentación, se observó que la mayoría de los sujetos en estudio realizaba 3 comidas establecidas al día (63\%), considerándose como tiempos irregulares de alimentación (omisión de una o más de las 4 comidas recomendadas). Se observó que el 100\% de la muestra consumía el almuerzo, seguido por la merienda (96\%) y el desayuno (84,6\%). Estos resultados son concordantes con la Encuesta Nacional de Consumo Alimentario 2014 (ENCA), que mostró que el tiempo de comida que consume la mayoría de los chilenos es el almuerzo (96\%), seguido por el desayuno $(90 \%)$ y la merienda $(80 \%)^{18}$. Cabe destacar que este patrón se mantiene en la mayoría de los sujetos estudiados, independiente de su estado nutricional. Además, se observó que el 86\% los universitarios consumen snacks, con un aporte energético promedio de $362 \pm 308$ kcal/día, representando en promedio el $21 \%$ de la ingesta calórica diaria.

En cuanto a los horarios de alimentación, se observó que el $84 \%$ de los individuos presentan horarios de alimentación irregulares, con intervalos entre comidas de más de 4 horas de ayuno en al menos una ocasión del día, y sin colaciones establecidas. Esta variable no se pudo comparar con los datos de la ENCA 2014, ya que en esa encuesta no se evaluaron los intervalos entre tiempos de comida.

En diversos estudios se ha evidenciado una relación inversa entre la ingesta del desayuno y el IMC en niños, adolescentes y adultos ${ }^{19,20,21}$. En este caso, el 44,6\% de los estudiantes tenía un consumo poco frecuente del desayuno ( $<5$ veces por semana). Aun así, no se logró establecer una relación de dependencia entre la ingesta del desayuno y el porcentaje de grasa corporal (datos no se muestran), al haber un alto porcentaje de sujetos normales con una ingesta poco frecuente de desayuno. Metodológicamente, al comparar nuestros resultados con estudios similares, es importante considerar diferencias al definir la variable de ingesta del desayuno, nuestra investigación estableció que los sujetos tenían hábito de desayuno cuando lo consumían con una frecuencia de 6 a 7 veces a la semana, mientras que otros estudios consideran éste como un hábito cuando presentaba una frecuencia de 5 a 7 veces a la semana, por intervalos de días, o de manera cualitativa ${ }^{10}$.

Con respecto a la distribución energética del desayuno de acuerdo al total diario, se observó que los hombres con exceso de grasa corporal tienen un \% de adecuación significativamente menor en comparación a los sujetos normales y a los que presentaron bajas reservas energéticas $(p=0.021)$ (Tabla 3). Pocos estudios han relacionado el aporte energético de este tiempo de comida o su adecuación en relación al aporte total diario con el estado nutricional. Se ha descrito que los individuos obesos tienden a consumir menor energía en el desayuno e incluso omitir este tiempo de comida $^{10}$, lo que es concordante a lo observado en hombres según porcentaje de grasa corporal.

En este estudio no se evaluó la calidad de cada macronutriente en este tiempo de comida (cantidad de fibra, complejidad de carbohidratos, tipo de ácidos grasos y fuente alimentaria de las proteínas), en contraste con las investigaciones mencionadas anteriormente, sin embargo, durante el proceso de recopilación de información de la encuesta alimentaria, se observó una preferencia por alimentos procesados y altos en grasa saturada, principalmente en sujetos obesos.

Por otro lado, se ha descrito que omitir el desayuno no solo podría influir en los hábitos alimentarios en el resto del día, sino que también en el estilo de vida de los individuos, como lo muestra el estudio de Keski-Rahkonen et al., donde se observó que los individuos que no consumían desayuno (adolescentes y adultos) presentaban un mayor consumo de alcohol, cafeína, tabaco, y menor actividad física ${ }^{19}$.

Con respecto a la asociación de tiempos y horarios de comida según porcentaje de grasa corporal, se encontró una relación de dependencia, donde el $89 \%$ de los sujetos con exceso de grasa corporal también refirieron tener horarios de comida irregulares $(p=0.044)$ (Tabla 5). Horarios de alimentación regulares podrían contribuir en la mantención de la homeostasis de las hormonas sintetizadas por el páncreas, regulando los niveles de glucosa en sangre y evitando la ansiedad ${ }^{22}$.

En relación a la ingesta energética diaria, se observa que los sujetos con exceso de peso no logran cubrir sus requerimientos energéticos según lo recomendado por FAO/OMS según sexo y estado nutricional (Tabla 4). Esta situación se puede deber a una sobreestimación de sus requerimientos energéticos de acuerdo al factor asignado por actividad física y también a una subestimación de la 
ingesta de alimentos referida por estos sujetos.

Si analizamos la distribución calórica por tiempo de comida, podemos observar que es en la merienda donde la ingesta energética supera los límites recomendados (20$25 \%$ ) en comparación con las demás comidas diarias para todos los estados nutricionales (Tabla 6). Por otro lado, los sujetos con sobrepeso y obesidad tienen una menor ingesta energética a lo recomendado en el desayuno, omiten la cena, desplazando la merienda a un horario más tardío (en promedio $21.00 \mathrm{hrs}$ ) e incorporando "snack" nocturnos (entre las 22.00 y 00.00hrs). Del total de la muestra según composición corporal, se observó una menor ingesta calórica en el almuerzo entre los sujetos con exceso de grasa corporal versus los normales. Por sexo, se encontraron diferencias significativas en hombres y mujeres, ya sea en la baja ingesta de energía del desayuno o durante almuerzo; respectivamente (datos no se muestran). Estudios postulan que tener una baja ingesta u omisión del desayuno, puede conducir a una mayor ingesta energética durante la merienda o cena, lo cual es concordante con estos resultados, además de generar inflamación de bajo grado y alterar la homeostasis de la glucosa ${ }^{23}$. Por otra parte, se ha descrito que la ingesta del desayuno está asociada a una mayor frecuencia de alimentación, lo cual podría aumentar la termogénesis de los alimentos, asociándose a un menor $\mathrm{IMC}^{24,25}$. El desayuno es considerado una parte importante de un estilo de vida saludable gracias a los hallazgos de diversos estudios observacionales que han reportado asociaciones entre el consumo de desayuno y beneficios para la salud, incluyendo mejor estado nutricional y menor riesgo cardiovascular ${ }^{27,28}$. Por otra parte, las recomendaciones respecto a este tiempo de comida han sido cuestionadas recientemente debido a la falta de evidencia que avale la ingesta de desayuno para promover la baja de peso y de grasa corporal en estudios clínicos randomizados ${ }^{29,30}$. Diferencias en la duración y diseño de las intervenciones, el aporte energético, en el aporte nutricional de distintos macronutrientes y/o calidad de estos, los sujetos en estudio (distintos grupos de edad, magros o con exceso de peso) podrían explicar la diversidad de resultados reportados en este tipo de estudios. En relación a la discrepancia de los hallazgos según tipo de estudio, será quizás necesario diferenciar lo que debe ser recomendado en término de hábitos alimentarios saludables, para la promoción y mantención de la salud en individuos aparentemente saludables a largo plazo y las recomendaciones para el tratamiento o manejo de exceso de peso en poblaciones en riesgo. En este sentido, y en relación a la población a la que está dirigida el presente estudio, más investigaciones respecto al consumo habitual del desayuno, tipos de alimentos incluidos versus los recomendados, con datos del tamaño de porciones y composición nutricional más detallada, junto con determinar posibles mecanismos, como variaciones en niveles de hormonas, apetito y/o saciedad; serán requeridos para determinar los efectos beneficiosos de la ingesta de desayuno habitual para promover la salud.
De acuerdo a los datos obtenidos en el presente estudio, se observó un alto consumo de "snacks". Los individuos obesos consumían 3 o más "snacks", principalmente durante la tarde-noche, con una mayor elección de snacks no saludables. Se ha descrito una relación entre horarios y distribución calórica de las comidas diarias, recomendándose una mayor ingesta al desayuno y una ingesta reducida durante las 18.00 a $21.00 \mathrm{hrs}^{5}$. En este caso, en los sujetos en estudio observamos que la mayor parte de su ingesta ocurría durante la tarde noche. Al respecto, aún no está claro qué porcentaje es el que se debiera consumir durante las primeras horas del día, un estudio indica que debiera ser un $50 \%$ durante el desayuno ${ }^{20}$, otro muestra beneficios en el estado nutricional cuando el $70 \%$ de la ingesta diaria se consume antes de las $12.00 \mathrm{pm}^{26}$, mientras que un tercer estudio recomienda consumir $<33 \%$ después de las $17.00 \mathrm{hrs}^{7}$. Estas investigaciones indican que, aparte del balance energético positivo como causa del exceso de peso, es fundamental la distribución de la ingesta energética durante el día.

El reducido tamaño de la muestra y que ésta haya sido no probabilística son las principales limitaciones de este estudio, junto con debilidades propias de un estudio transversal. En relación a las fortalezas, el estudio de la distribución energética durante el día, el aporte nutricional de desayuno y horarios de alimentación son aspectos que han sido poco estudiados en estudiantes universitarios y en población chilena en general. Su posible relación con el estado nutricional podría ser de utilidad para establecer estrategias de promoción de salud y prevención relativos a la distribución de comidas durante el día en este grupo de adultos jóvenes.

\section{CONCLUSIONES}

Considerando los resultados de la presente investigación se puede establecer una relación de dependencia entre los horarios de alimentación y el porcentaje de grasa corporal. Se observó que los sujetos obesos presentaban una menor ingesta al desayuno y al almuerzo en relación a lo recomendado; y un mayor consumo de snack no saludables, en horarios nocturnos. En relación a la composición nutricional del desayuno, esta no se asoció con el porcentaje de grasa corporal ni con el estado nutricional según IMC de los pacientes, pero sí cuanto el desayuno representaba de la ingesta energética total diaria Esto, evidencia que en población universitaria se debe mejorar la distribución energética durante el día, privilegiando un mayor consumo energético durante el desayuno/almuerzo y promoviendo horarios de alimentación estables, con 4 comidas al día y evitando el consumo intempestivo de snacks procesados durante la tarde-noche, con la finalidad de contribuir a mejorar el estado nutricional y composición corporal de estudiantes universitarios sedentarios, la cual ha empeorado en los últimos años, haciendo que este grupo tenga un alto riesgo de desarrollar enfermedades crónicas no 
transmisibles a mediano y largo plazo. En este contexto, los estudiantes universitarios debiesen ser considerados un grupo objetivo de intervención nutricional, para así evitar que estos hábitos alimentarios asociados a un exceso de grasa corporal se perpetúen en la adultez.

Agradecimientos. A los participantes de este estudio por su colaboración desinteresada.

\section{BIBLIOGRAFÍA}

1. Cervera Burriel Faustino SUR, Vico García Cruz, Milla Tobarra Marta, García Meseguer María José. Hábitos alimentarios y evaluación nutricional en una población universitaria. Nutr Hosp 2013; 28(2): 438-446.

2. Arroyo Izaga M, Rocandio Pablo, A. M.a, Ansotegui Alday, L., Pascual Apalauza, E., Salces Beti, I., Rebato Ochoa, E. Calidad de la dieta, sobrepeso y obesidad en estudiantes universitarios. Nutr Hosp 2006; 21: 673-679.

3. Alcacera MA, Marques-Lopes I, Fajo-Pascual M, Foncillas $J P$, Carmona-Torre F, Martinez-Gonzalez MA. Alcoholic beverage preference and dietary pattern in Spanish university graduates: the SUN cohort study. Eur I Clin Nutr 2008; 62(10): 1178-1186.

4. Garaulet M, Gomez-Abellan P. Timing of food intake and obesity: a novel association. Physiol Behav 2014; 134: 44-50.

5. Jakubowicz D, Barnea M, Wainstein J, Froy O. High caloric intake at breakfast vs. dinner differentially influences weight loss of overweight and obese women. Obesity (Silver Spring, Md) 2013; 21(12): 2504-2512.

6. Shafiee G, Kelishadi R, Qorbani M, Motlagh ME, Taheri $M$, Ardalan G, et al. Association of breakfast intake with cardiometabolic risk factors. J Pediatr (Rio J) 2013; 89(6): 575-582.

7. Wang JB, Patterson RE, Ang A, Emond JA, Shetty N, Arab L. Timing of energy intake during the day is associated with the risk of obesity in adults. I Hum Nutr Diet 2014;27 Supp/ 2: 255-262.

8. Garaulet M, Sanchez-Moreno C, Smith CE, Lee YC, Nicolas $F$, Ordovas JM. Ghrelin, sleep reduction and evening preference: relationships to CLOCK 3111 T/C SNP and weight loss. PloS one 2011; 6(2): e17435.

9. Garaulet M, Esteban Tardido A, Lee YC, Smith CE, Parnell LD, Ordovas JM. SIRT1 and CLOCK 3111T> C combined genotype is associated with evening preference and weight loss resistance in a behavioral therapy treatment for obesity. Int J Obes (Lond.) 2012; 36(11): 1436-1441.

10. Cho S, Dietrich M, Brown CJ, Clark CA, Block G. The effect of breakfast type on total daily energy intake and body mass index: results from the Third National Health and Nutrition Examination Survey (NHANES III). I Am Coll Nutr 2003; 22(4): 296-302.

11. Rabinovitz HR, Boaz M, Ganz T, Jakubowicz D, Matas Z, Madar Z, et al. Big breakfast rich in protein and fat improves glycemic control in type 2 diabetics. Obesity (Silver Spring). 2014; 22(5): E46-54.

12. Gallagher D, Heymsfield SB, Heo M, Jebb SA, Murgatroyd $P R$, Sakamoto $Y$. Healthy percentage body fat ranges: an approach for developing guidelines based on body mass index. Am J Clin Nutr 2000; 72(3): 694-701.

13. WHO. Human energy requirements: report of a Joint FAO/ WHO/UNU Expert Consultation. Food Nutr Bull 2005;
26(1): 166.

14. Jury G, Urteaga C, R CU, Taibo M, Alimentos UdCldNyTdl. Porciones de intercambio y composición química de los alimentos de la pirámide alimentaria chilena. Santiago: LOM ediciones; 1997.

15. Ministerio de Salud. Encuesta Nacional de Salud 20162017. Disponible en: http://www.minsal.cl/wp-content/ uploads/2017/11/ENS-2016-17_PRIMEROS-RESULTADOS. pdf.

16. Carrasco F, Reyes E, Rimler O, Rios F. Exactitud del índice de masa corporal en la predicción de la adiposidad medida por impedanciometría bioeléctrica. Arch Latinoam Nutr 2004; 54(3): 280-286.

17. Martín Moreno V, Gómez Gandoy JB, Antoranz González MJ. Medición de la grasa corporal mediante impedancia bioeléctrica, pliegues cutáneos y ecuaciones a partir de medidas antropométricas. Análisis comparativo. Rev Esp Salud Pública 2001; 75: 221-236.

18. MINSAL. Encuesta Nacional de Consumo Alimentario ENCA. Universidad de Chile, Gobierno de Chile; 2014. p. 11-34.

19. Keski-Rahkonen A, Kaprio J, Rissanen A, Virkkunen M, Rose RJ. Breakfast skipping and health-compromising behaviors in adolescents and adults. Eur J Clin Nutr 2003; 57(7): 842-853.

20. Timlin MT, Pereira MA, Story $M$, Neumark-Sztainer D. Breakfast eating and weight change in a 5-year prospective analysis of adolescents: Project EAT (Eating Among Teens). Pediatrics 2008; 121(3): e638-645.

21. Thompson-McCormick JJ, Thomas JJ, Bainivualiku A, Khan AN, Becker AE. Breakfast skipping as a risk correlate of overweight and obesity in school-going ethnic Fijian adolescent girls. Asia Pac J Clin Nutr 2010; 19(3): 372-382.

22. Vieira E, Burris TP, Quesada I. Clock genes, pancreatic function, and diabetes. Trends Mol Med 2014; 20(12): 685-693.

23. Nas A, Mirza N, Hagele F, Kahlhofer I, Keller J, Rising R, et al. Impact of breakfast skipping compared with dinner skipping on regulation of energy balance and metabolic risk. Am J Clin Nutr 2017; 105(6): 1351-1361.

24. Drummond S, Crombie N, Kirk T. A critique of the effects of snacking on body weight status. Eur J Clin Nutr 1996; 50(12): 779-783.

25. Martin A, Normand S, Sothier M, Peyrat J, Louche-Pelissier C, Laville M. Is advice for breakfast consumption justified? Results from a short-term dietary and metabolic experiment in young healthy men. Br J Nutr 2000; 84(3): 337-344.

26. Keim NL, Van Loan MD, Horn WF, Barbieri TF, Mayclin PL. Weight loss is greater with consumption of large morning meals and fat-free mass is preserved with large evening meals in women on a controlled weight reduction regimen. I Nutr 1997; 127(1): 75-82.

27. Odegaard AO, Jacobs DR, Steffen LM, Van Horn L, Ludwig $D S$, Pereira MA. Breakfast frequency and development of metabolic risk. Diabetes Care 2013; 36: 3100-3106.

28. Deshmukh-Taskar P, Nicklas TA, Radcliffe JD, O'Neil CE, Liu Y. The relationship of breakfast skipping and type of breakfast consumed with overweight/obesity, abdominal obesity, other cardiometabolic risk factors and the metabolic syndrome in young adults. The National Health and Nutrition Examination Survey (NHANES): 1999-2006. Public Health Nutrition 2013; 16(11): 2073-2082.

29. Leidy HI, Ortinau LC, Douglas SM, Hoertel HA. Beneficial 
effects of a higher-protein breakfast on the appetitive, hormonal, and neural signals controlling energy intake regulation in overweight/obese, "breakfast- skipping," late-adolescent girls. Am J Clin Nutr 2013; 97: 677-688.
30. Brown AW, Bohan Brown MM, Allison DB. Belief beyond the evidence: using the proposed effect of breakfast on obesity to show 2 practices that distort scientific evidence. Am J Clin Nutr 2013; 98: 1298-1308. 